\title{
Commentaries
}

\section{Helicobacter pylori and dyspepsia: trick or treat?}

Clinicians working in both primary and secondary care still do not have evidence-based guidance on the management of dyspepsia which is both clinically effective and cost-efficient. Perhaps we never will. Moving therapeutic targets often generate questions faster than researchers can answer them. Nevertheless, we do need better information about the inter-relations between dyspepsia, Helicobacter pylori infection, $H$ pylori eradication, and endoscopy. The paper by Heaney et al (see page 186) provides a good deal of information, but also raises further questions about alternative strategies for managing dyspepsia.

The background is familiar. Until $H$ pylori burst on to the scene, there was some controversy about empirical therapy versus early endoscopy in the initial management of dyspepsia, but the availability of accurate diagnostic and effective therapeutic methods in $H$ pylori management means that clinicians can now choose from two further strategies-(1) test and endoscope and (2) test and treat. In the first of these the $H$ pylori test is used as a "screen" for endoscopy, with $H$ pylori positive patients undergoing endoscopy and $H$ pylori negative patients treated as if they have non-ulcer dyspepsia. Patel and colleagues ${ }^{1}$ claimed that this approach would reduce the need for endoscopy by about $40 \%$. The test and treat strategy, conversely, implies that uninvestigated dyspeptic patients (without alarm symptoms) should be tested for $H$ pylori infection using the most accurate method available and those testing positive should undergo $H$ pylori eradication therapy whereas those testing negative should be diagnosed as non-ulcer dyspepsia (and, presumably, gastrooesophageal reflux disease as well).

Most of the information that we have had up until now, as Heaney and colleagues point out, has come not from prospective randomised trials, but from computer simulations of various kinds. ${ }^{2}$ Even this shadowy area of research has generated sparky controversy, with some groups concluding that there was little to choose in clinical or cost terms between the various treatments and others claiming therapeutic gain or cost savings, particularly from the test and treat approach. ${ }^{4}$ Heaney's group are probably right in claiming that this is the first prospective randomised trial comparing a test and treat strategy with endoscopic "business as usual".

One of the attractions of Heaney et al's paper is that it dips its toe fairly determinedly in the potentially murky waters of health services research. As well as measuring conventional parameters including endoscopic findings, $H$ pylori breath test results and symptom scores, the authors also used the SF36 to examine changes in quality of life and the Crown Crisp index to examine personality traits, although the latter does not seem to have generated any interesting information. Patients were followed for 12 months, which was a welcome extension of the normal length of follow up in $H$ pylori eradication trials, but the study design could have been further strengthened by the incorporation of a formal health economic evaluation.

The results make interesting and stimulating reading. They are fairly persuasive that, taken in isolation from the possible disbenefits of eradication of $H$ pylori and the overuse of antibiotics and community antibiotic resistance, a test and treat strategy is clinically appropriate and resource-sparing. Their conclusions are bolstered by the finding, presumably a surprise to the authors, of an excess of symptomatic improvement in the test and treat group over the endoscopy group. This observation is, incidentally, at odds with the received wisdom that a normal upper gastrointestinal endoscopy leads to reductions in anxiety, severity of symptoms and health care resource use. ${ }^{56}$ Other unexpected side effects of this paper are some useful information about responses to triple therapy given at various stages in the management of $H$ pylori positive patients and also the sensitivity to change of the SF36 in this group of patients without severe illness.

The authors provide an important caveat, which is that these results were obtained at a secondary referral centre and should not be extrapolated to primary care. They recommend the evaluation of a similar strategy in this setting. The results of a comparable controlled trial in primary care, which included a health economic evaluation, are currently in press, and broadly support the conclusions of Heaney's group, adding to existing evidence of clinical effectiveness information about the cost-savings associated with empirical $H$ pylori eradication in the uninvestigated dyspeptic patient. $^{7}$

However, whether or not these findings should be translated into clinical practice depends on the answers to a number of research questions. Firstly, will we do a disservice to patients without duodenal ulcer disease when we eradicate their $H$ pylori? Uncertainties still exist about the value of $H$ pylori eradication in non-ulcer dyspepsia, ${ }^{8}$ particularly in primary care patients in whom little research has been done. There are also concerns about the long term risks of gastro-oesophageal reflux disease and cancer after $H$ pylori eradication. ${ }^{9}$ Prospective randomised studies in primary care with adequate length of follow up are required to settle these points. Secondly, does the "unnecessary" use of triple therapy (i.e. in non-ulcer $H$ pylori positive patients) lead to unacceptable consequences to individuals (in terms of side effects of therapy) or to the community (in terms of increasing levels of antibiotic resistance)? Compared with the widespread use of these drugs in primary care to treat respiratory, gastrointestinal and pelvic symptoms and infections, the contribution of triple therapy is likely to be minimal, although clear information is still needed on this point. Thirdly, will the increasingly widespread use of a test and treat approach, particularly when it is based on suboptimal methods of detecting $H$ pylori infection (such as some currently available near patient serology kits) lead to a less critical and potentially hazardous evaluation of dyspeptic patients? Perhaps, only time will tell, but $H$ pylori testing must not be allowed to take the place of careful clinical assessment.

ROGER JONES

Department of General Practice and Primary Care, Guy's, King's and St Thomas's School of Medicine, 5 Lambeth Walk,

London SE11 6SP, UK

1 Patel P, Khulusi S, Mendall MA, et al. Prospective screening of dyspeptic patients by Helicobacter pylori serology. Lancet 1995;346:1315-18. 
2 Fendrick AM, Chernew ME, Hirth RA, et al. Immediate endoscopy or initial Helicobacter pylori serological testing for suspected peptic ulcer disease: estimating cost effectiveness using decision analysis. Yale f Biol Med 1996;69:187-95.

3 Silverstein MD, Petterson T, Talley NJ. Initial endoscopy or empirical therapy with or without testing for Helicobacter pylori for dyspepsia: a decision analysis. Gastroenterology 1996;110:72-83.

4 Tait CL, Jones RH. Decision analytic modelling of dyspepsia management strategies [abstract]. Gastroenterology 1997;112:A45.

5 Jones R. What happens to patients with non-ulcer dyspepsia after endoscopy? Practitioner 1988;232:75-8.
6 Hungin AP, Thomas PR, Bramble MG, et al. What happens to patients after open-access gastroscopy? An outcome study from general practice. $\mathrm{Br} \mathcal{F}$

7 Jones R, Tait C, Sladen G, et al. A trial of a test and treat strategy for Helicobacter pylori positive patients in general practice. Int $\mathcal{F}$ Clin Pract (in press).

8 Laheij RJ, Jansen JB, van de Lisdonk EH, et al. Symptom improvement through eradication of Helicobacter pylori in patients with non ulcer dyspepsia. Aliment Pharmacol Ther 1996;10:843-50.

9 Labenz J, Tillenburg B, Peitz U, et al. Long term consequences of Helicobacter pylori eradication: clinical aspects. Scand $\mathcal{f}$ Gastroenterol 1996; (suppl 215):111-15.

\section{The pill: safe sex and Crohn's disease?}

Epidemiological data suggest that there is a small increase in the risk of Crohn's disease in women who use the combined oral contraceptive pill, ${ }^{1-3}$ the relative risk being about twofold. On this basis, many physicians advise women with Crohn's disease not to use this form of contraception. Is there really sufficient evidence to justify this advice? Unlike smoking, ${ }^{1}$ there is no clear evidence of an association between oral contraceptive use and the prevalence of Crohn's disease. The available data are confounded by problems with study design and also the influence of smoking, as some studies have shown that smokers are more likely to use oral contraception. There is little information on whether the type of oral contraception may explain some of the differences observed, in particular the oestrogen dose and the length of use.

Patients with active inflammatory bowel disease are at increased risk of developing thromboembolic disease and there are also theoretical reasons to suppose that the pill might precipitate or exacerbate Crohn's disease. High dose oral contraceptives, containing more than $50 \mu \mathrm{g}$ ethyloestradiol, may induce prothrombotic changes in both coagulation and fibrinolytic enzyme systems, thereby predisposing to the formation of microthrombi. This would fit with the microvascular hypothesis of the pathogenesis of Crohn's disease. ${ }^{4}$ Early reports that the withdrawal of oral contraception was associated with long term remission in patients with Crohn's colitis could be used to support this hypothesis. ${ }^{5}$ For many years, however, low dose oral contraceptive preparations containing $30 \mu \mathrm{g}$ ethinyloestradiol or less have usually been prescribed, and although these do activate coagulation and fibrinolysis, the effects are less pronounced. The influence of the type and dose of progestogen and the length of time for which the pill has been taken as well as other factors such as combination with smoking and the increasing recognition of inherited causes of thrombophilia, may also be of relevance. ${ }^{6}$

Smoking has been associated with increased severity in terms of disease activity in patients with Crohn's disease. A number of small studies have also looked at whether oral contraceptive use is associated with increased disease severity and activity. In this issue, Cosnes et al (see page 218) report the results of a carefully performed, prospective observational study examining the effect of oral contraceptive use on the clinical course of Crohn's disease in a cohort of 331 women aged 16-50 who were followed for up to 18 months. Of the women, 134 (40.5\%) used oral contraceptives, defined as taking the pill during the month before inclusion into the study. The oral contraceptive users were younger, with a median age of 28 years compared with a median of 32 for the non-users; the nonusers had a longer duration of disease: 78 compared with
52 months. There were no differences in smoking habits, disease activity in the previous year, current disease activity, or other medication. Most of those using oral contraceptives were taking a $30 \mu \mathrm{g}$ ethinyloestradiol preparation but there is no information about the duration of oral contraceptive use or whether non-users had taken oral contraceptives in the past. It is possible that they had as they were older than the current users. The relapse rate during the study period was similar: $46 \%$ in users compared with $43 \%$ in the non-users. No thrombotic events were observed during the study period.

These results differ from another recently published prospective study from Canada. ${ }^{8}$ Timmer et al reported the influence of smoking, oral contraceptive use and other factors influencing Crohn's disease relapse. Their results in a small cohort of 88 women who were randomised to placebo in a controlled trial of mesalamine in the prevention of Crohn's disease relapse, suggest that women who had ever used oral contraceptives had a threefold increase in relapse rate. It is difficult to explain how past use of the pill could affect current relapse rates, although it is possible to postulate that these women had taken high dose pills in the past and this could have been an aetiological factor in their disease in the first place. There are methodological problems with using these placebo treated women and in Timmer et al's study current pill users did not in fact have different relapse rates from non-users, unless the current users were combined with women who had ever used the pill, making this difficult to attribute to an effect of oral contraception on relapse.

Cosnes et al's study shows that despite the weak association between oral contraceptive use and the development of Crohn's disease, in women with Crohn's disease the use of low dose oral contraceptive is not associated with increased disease activity compared with non-users. The power of this study was adequate to detect the effect of smoking and so this would concur with evidence from other investigators that the use of the low dose pill is not associated with increased Crohn's disease recurrence. ${ }^{8}$

So, how should we advise our patients on the basis of this new evidence? Strong advice not to smoke is obviously essential and the pill does not seem to be an appropriate form of contraception for smokers. Women with quiescent or mildly active Crohn's disease who do not smoke or have any other risk factors for thromboembolic disease may, however, make an informed decision after discussion with their physician whether to start or continue taking the pill.

Whipps Cross Hospital, Whipps Cross Road,

E M ALSTEAD Leytonstone, London E11 1NR, UK

1 Kay CR, Logan RFA. Oral contraception, smoking and inflammatory bowel disease-findings in the Royal College of General Practitioners Oral Contraception Study. Int $\mathcal{F}$ Epidemiol 1989;18:105-7.

2 Godet PG, May GR, Sutherland LR. Meta-analysis of the role of oral contraceptive agents in inflammatory bowel disease. Gut 1995;37:668-73.

3 Boyko EJ, Theis MK, Vaughan TL, et al. Increased risk of IBD associated with oral contraceptive use. Am $\mathcal{F}$ Epidemiol 1994;140:268-78. 
4 Wakefield AJ, Sawyerr AM, Dhillon AP, et al. Pathogenesis of Crohn's disease: Multifocal gastrointestinal infarction. Lancet 1989;ii: 1057-62.

5 Vessey $\mathrm{M}$, Jewell $\mathrm{D}$, Smith A, et al. Chronic inflammatory bowel disease, cigarette smoking and use of oral contraceptives-findings in a large cohort study of women of childbearing age. BMf 1986;292:1101-3.

6 Norris LA, Bonnar J. The effect of oestrogen dose and progestogen type on hemostatic changes in women taking low dose oral contraceptives. Brf Obstet Gynaecol 1996;103:261-7.

7 Timmer A, Sutherland LR, Martin F. Canadian mesalamine for remission of Crohn's disease study group. Gastroenterology 1998;114:1143-50.

8 Sutherland LR, Ramcharan S, Bryant H, et al. Effect of oral contraceptive use on reoperation following surgery for Crohn's disease. Dig Dis Sci 1992; 37:1377-82.

\section{Gall bladder and bowel: the links multiply}

Penning et al's paper is a brain-teaser (see page 264). It clearly shows is that, in the laboratory, the gall bladder behaves abnormally in patients with chronic constipation. What is much less clear, but very intriguing, is what the findings mean. Recently, the state of the gall bladder in patients with constipation has been attracting attention because of growing evidence that cholesterol gallstones can result from slow intestinal transit. ${ }^{1-3}$ Slow transit allows the colon to absorb excessive amounts of bacterially degraded substances, including the toxic bile acid, deoxycholic acid (DCA). When DCA reaches the liver, it makes it secrete more cholesterol into bile and when it reaches the gall bladder it hastens the crystallisation of cholesterol. ${ }^{2}{ }^{4}$ So, patients with slow transit constipation should be at high risk of gallstones. Puzzle number one is that the patients in Penning et al's study seem to have escaped gallstones (although this is not explicitly stated).

But was their gall bladder motility defect likely to lead to gallstones? Impaired gall bladder emptying is certainly a feature of patients with cholesterol gallstones. ${ }^{5}$ Indeed, it is believed to be a key factor in stone pathogenesis, if only because it favours the growth and retention of calculi within the gall bladder. Latest thinking is that the gall bladder of patients with gallstones may be a poor contractor because its wall is infiltrated with cholesterol, and perhaps inflamed by the high DCA concentrations in the bile inside it. ${ }^{46}$ It is tempting to welcome Penning et al's findings as fitting in with this scheme, the slow transit of their patients having led to bile toxic to the gall bladder. However, there is a snag with this interpretation. In the characteristic motility defect of patients with gallstones the resting volume of the gall bladder is large, ${ }^{4}$ whereas in these constipated patients, the resting volume was smaller than normal - a finding which is unusual and curious.

Another difficulty in comparing these new data with published ones is the method used to demonstrate impaired gall bladder emptying, namely sham feeding. The results cannot be extrapolated to the natural stimulus of eating a meal. The response to cholecystokinin - the mediator of food induced contraction-was normal; indeed, in another study of constipated patients (who also had irritable bowel syndrome (IBS)), the response to a cholecystokinin analogue was actually greater than normal. ${ }^{7}$ So at least we can be sure of one thing - the gall bladder muscle is not to blame. Is it a problem of understimulation? Because the gall bladder's response to sham feeding involves vagal cholinergic stimulation, Penning et al conclude that failure to respond is a sign of vagal neuropathy. This sounds logical but would be more convincing if tests of vagal function had been carried out and a relation shown between these and the gall bladder dysmotility.

Penning et al ignore the possibility of psychological factors. The response of the gastrointestinal tract to sham feeding is called the cephalic phase and it involves the higher centres of the brain. Having to chew and spit out a meal cannot be a pleasant experience. Suppose that some participants found it positively disgusting, would their gastrointestinal tracts, including their gall bladders, have reacted normally? The emotional reaction of the patients might have been stronger than that of the normal controls. Almost by definition, patients are anxious people (why else do they go to the doctor?) and so more liable to strong emotional reactions. Constipation can itself be a somatic expression of psychic distress ${ }^{8}$ (though patients with functional gastrointestinal disorders who reach specialists are likely to deny their distress ${ }^{9}$ ).

In discussing their findings, Penning et al have avoided the biopsychosocial approach but, more conventionally perhaps, have suggested that constipated people have a widespread smooth muscle disorder encompassing the gall bladder as well as the intestine (not a new idea ${ }^{3}$. This is the language used in the past about IBS but the evidence that gall bladder function is abnormal in IBS, protean though that condition is, is inconsistent. ${ }^{10}$ Also, the trend in IBS is towards larger fasting volumes, ${ }^{11}$ not smaller as in Penning et al's patients. A better analogy might be with functional dyspepsia, where impaired relaxation of the stomach seems to be a feature ${ }^{12}$ because impaired relaxation of the gall bladder could explain the small fasting volume in Penning et al's patients.

This study, like many, raises more questions than it answers. It reminds us how little we understand the behaviour of our digestive systems. Functional disorders are difficult to study but study them we must because, more and more, these are the disorders which trouble the patients who face us in our clinics. And every study of a functional disorder should take account of psychosocial factors.

Claverham House,

$\mathrm{K}$ W HEATON

Streamcross,

Claverham,

N. Somerset BS49 4QD, UK

1 Heaton KW, Emmett PM, Symes CL, et al. An explanation for gallstones in normal-weight women: slow intestinal transit. Lancet 1993;341:8-10.

2 Dowling RH, Veysey MJ, Pereira SP, et al. Role of intestinal transit in the pathogenesis of gallbladder stones. Can f Gastroenterol 1997;11:57-64.

3 van Erpecum KJ, van Berge-Henegouwen GP. Gallstones: an intestinal disease? Gut 1999;44:435-38.

4 Portincasa $\mathrm{P}$, van de Meeberg $\mathrm{P}$, van Erpecum KJ, et al. An update on the pathogenesis and treatment of cholesterol gallstones. Scand $\mathcal{f}$ Gastroenterol 1997;32:60-9.

5 Jazrawi RP, Pazzi P, Petroni ML, et al. Postprandial gallbladder motor function: refilling and turnover of bile in health and in cholelithiasis. Gastronterology 1995;109:582-91.

6 Apstein MD, Carey MC. Pathogenesis of cholesterol gallstones: a parsimonious hypothesis. Eur F Clin Invest 1996;26:343-52.

7 Kellow JE, Miller LJ, Phillips SF, et al. Altered sensitivity of the gallbladder to cholecystokinin octapeptide in irritable bowel syndrome. Am f Physiol 1987;253:G650-5.

8 Devroede G, Girard G, Bouchoucha M, et al. Idiopathic constipation by colonic dysfunction. Dig Dis Sci 1989;34:1428-33.

9 Heaton KW, Thompson WG, Smyth GT, et al. When and why do general practitioners refer IBS patients to gastroenterologists [abstract]? Gut 1998; 42:A61

10 McKee DP, Quigley EMM. Intestinal motility in irritable bowel syndrome: Is IBS a motility disorder? Part 2. Motility of small bowel, esophagus, stomach and gall-bladder. Dig Dis Sci 1993;38:1773-82.

11 Sood GK, Baijal SS, Lahoti D, et al. Abnormal gallbladder function in patients with irritable bowel syndrome. Am f Gastroenterol 1993;88:1387-90.

12 Troncon LEA, Thompson DG, Ahluwalia NK, et al. Relations between upper abdominal symptoms and gastric distension abnormalities in upper abdomotility like functional dyspepsia and after vagotomy. Gut 1995;37:1722 . 


\section{Cyclosporin and chronic pancreatitis: a supermodel?}

Most patients with acute pancreatitis develop their disease in association with either biliary tract stones or abuse of ethanol, whereas chronic pancreatitis is most commonly the result of prolonged ethanol abuse. Attempts to study the pathogenesis and pathophysiology of these inflammatory conditions have been hampered by the relative inaccessibility of the pancreas to study in humans, as well as the difficulty in identifying appropriate patients during the earliest stages of their disease.

Over the past two decades, several models of acute pancreatitis induced in laboratory animals have been developed..$^{1-3}$ Use of these models has permitted the performance of studies that have advanced our understanding of the early cellular events that underlie the development of acute pancreatitis. ${ }^{4}$ Unfortunately, similar progress in the area of chronic pancreatitis has not been made, mainly because no good models of that disease have been developed.

The hallmarks of chronic pancreatitis are the combined presence of chronic inflammatory changes, pancreatic fibrosis, and loss of pancreatic acinar cell mass. Several recent reports have suggested that the cytokine transforming growth factor (TGF) $\beta 1$ may play an important role in regulating these events in the pancreas and in other organs including the liver. Evidence supporting a role for TGF- $\beta 1$ includes the following observations: intrapancreatic expression of TGF- $\beta 1$ is increased in clinical chronic pancreatitis ${ }^{5}$; administration of TGF- $\beta 1$ to animals during acute pancreatitis promotes pancreatic fibrosis ${ }^{6}$; transgenic mice that overexpress TGF- $\beta 1$ in the pancreas develop pancreatic fibrosis ${ }^{7}$; and administration of anti-TGF- $\beta 1$ antibodies during acute pancreatitis in animals reduces extracellular matrix formation. ${ }^{8}$ The prevailing dogma suggests that TGF- $\beta 1$ promotes fibrogenesis by (a) favouring the proliferation and/or activation of collagen forming stellate cells (i.e. myofibroblasts) in the pancreas and other tissues ${ }^{9}$ and $(b)$ increasing the production of collagenase inhibitors. ${ }^{10}$

The immunosuppressant cyclosporin has been shown to stimulate TGF- $\beta 1$ production. ${ }^{11}$ In this issue, Vaguero et al (see page 269) report that administration of cyclosporin to rats increases TGF- $\beta 1$ levels in the pancreas. More importantly, however, they show that administration of cyclosporin to rats subjected to repeated episodes of secretagogue induced acute pancreatitis results in the development of chronic pancreatitis, characterised by the loss of pancreatic mass, proliferation of intrapancreatic myofibroblasts, increase in pancreatic hydroxyproline (i.e. collagen) content, development of pancreatic fibrosis, and appearance of a chronic inflammatory cell infiltrate within the pancreas.

These observations are of potential importance because they suggest that administration of cyclosporin to animals during experimental acute pancreatitis could provide the model of chronic pancreatitis which is so badly needed for studies probing the mechanisms underlying that disease. Clearly, the clinical relevance of the model described by Vaguero et al can be questioned as there is no reason to believe that clinical chronic pancreatitis is related to the combined effects of supramaximal secretagogue stimulation and exposure to cyclosporin. Similarly, there is no evidence that patients given cyclosporin for immunosuppression after non-pancreatic transplantation develop chronic pancreatitis and, in those given cyclosporin after pancreas transplantation, pancreatic atrophy, when it occurs, probably reflects rejection rather than chronic pancreatitis.

At the same time, however, it must be pointed out that the effective use of an animal model of disease is not dependent upon that model's recapitulation of the clinical events believed to be critical to the development of the disease. There are many examples of important insights gained using animal models of disease which are induced by interventions that may be, themselves, clinically irrelevant. Indeed, the most commonly used models of acute pancreatitis involve either administration of a choline deficient ethionine supplemented diet to young female mice $^{1}$ or the exposure of rodents to a supramaximally stimulating dose of the cholecystokinin analogue caerulein. ${ }^{2}$ Neither of these models would pass the "clinical relevance" test as few if any patients develop acute pancreatitis as the result of either ingesting an ethionine containing diet or being exposed to a supramaximally stimulating dose of a secretagogue. Yet both models have permitted performance of many studies that have elucidated basic cellular events that are critical to the development of acute pancreatitis.

It should be hoped that the model described by Vaguero et al will be similarly exploited for studies directed at elucidating early critical events in chronic pancreatitis. In this regard, evaluating the effects of cyclosporin administration during other forms of acute pancreatic injury might indicate whether the effects seen by Vaguero et al in the caerulein model are specific to that model or, alternatively, characteristic of pancreatic injury regardless of its cause. Similarly use of this model in transgenic or knockout mouse strains could permit dissection of the events which couple TGF- $\beta 1$ overexpression to chronic pancreatitis.

Professor of Surgery

M L STEER

Harvard Medical School and

Beth Israel Deaconess Medical Centre,

330 Brookline Avenue,

Boston, MA 02215, USA

1 Lombardi B, Estes LW, Longnecker DW. Acute hemorrhagic pancreatitis (massive necrosis) with fat necrosis induced in mice by DL-ethionine fed with a choline-deficient diet. Am F Pathol 1975;79:465-80.

2 Lampel M, Kern J. Acute interstitial pancreatitis in the rat induced by excessive doses of a pancreatic secretagogue. Virchows Arch 1977;373:97117.

3 Aho HJ, Koskensalo SM, Nevalainen TJ. Experimental pancreatitis in the rat. Sodium taurocholate-induced acute hemorrhagic pancreatitis. Scand $\mathcal{F}$ Gastroenterol 1980;15:411-16.

4 Steer ML. Frank Brooks Memorial Lecture. The early intra-acinar cell events which occur during acute pancreatitis. Pancreas 1998;17:31-7.

5 Van Laethem JL, Deviere J, Resibois A, et al. Localization of transforming growth factor $\beta 1$ and its latent binding protein in human chronic pancreatitis. Gastroenterology 1995;108:1873-81.

6 Van Laethem JL, Robberecht P, Resibois A, et al. Transforming growth factor $\beta$ promotes development of fibrosis after repeated courses of acute pancreatitis in mice. Gastroenterology 1996;110:576-82.

7 Lee MS, Gu D, Feng L, et al. Accumulation of extracellular matrix and developmental dysregulation in the pancreas by transgenic production of transforming growth factor-beta 1. Am f Pathol 1995;147:42-52.

8 Menke A, Yamaguchi H, Gress TM, et al. Extracellular matrix is reduced by inhibition of transforming growth factor $\beta 1$ in pancreatitis in the rat. Gastroenterology 1997;113:295-303.

9 Bachem MG, Sell KM, Melchior R, et al. Tumor necrosis factor alpha $(\mathrm{TNF} \alpha)$ and transforming growth factor $\beta 1$ (TGF $\beta 1$ ) stimulate fibronectin synthesis and the transdifferentiation of fat-storing cells in the rat liver into myofibroblasts. Virchows Arch 1993;63:123-30. 
10 Edwards DR, Murphy G, Reynolds JJ, et al. Transforming growth factor beta modulates the expression of collagenase and metalloproteinase inhibibeta modulates the expression of
tor. $E M B O \mp 1987 ; 6: 1899-904$.
11 Khanna A, Kapur S, Sharma VK, et al. In vivo over-expression of transforming growth factor- $\beta 1$ in humans; stimulation by cyclosporin. Transplant Proc 1997;29:284.

\section{$\mathrm{HBV}+\mathrm{HCV}=\mathrm{HCC} ?$}

Most people infected with hepatitis C virus (HCV) develop a chronic infection with persistent viraemia and are at risk of progressive liver damage. However, the outcome of chronic HCV infection is extremely variable and is influenced by many factors, including HCV genotype, $\mathrm{HCV}$ viral load, route of infection, age at infection, sex, and alcohol consumption. Ultimately, it is the progression to cirrhosis that is the key determinant of both morbidity and mortality ${ }^{1}$ and a better understanding of additional influences likely to promote this development is needed.

Both hepatitis B virus (HBV) and $\mathrm{HCV}$ are transmitted parenterally and coinfection is not uncommon, particularly in intravenous drug users and in countries with a high prevalence of $\mathrm{HBV}$ infection. ${ }^{2}$ Coinfection with evidence of chronic HBV and HCV seems to result in more severe liver disease than either infection alone, ${ }^{3}$ with an increased risk of liver cancer ${ }^{2}$ and probably an increased risk of fulminant hepatitis when superinfection with $\mathrm{HCV}$ occurs on the background of chronic HBV. ${ }^{4}$

The presence of hepatitis B surface and/or hepatitis B core antibodies (anti-HBs and anti-HBc) in the absence of hepatitis B surface antigen ( $\mathrm{HBs} A g$ ) is generally taken to indicate resolution of infection and provides evidence of previous HBV infection. However, it is becoming increasingly recognised that low levels of HBV DNA can still be found in serum, circulating lymphocytes and in the liver of many patients with apparently self-limited HBV infection years after clinical recovery from acute HBV infection. ${ }^{5}$ In keeping with this, transmission of HBV infection has been reported following transfusion of blood $^{6}$ or transplantation of livers from HBsAg negative, anti-HBc positive donors. ${ }^{7}$

It is not clear whether evidence of previous, rather than chronic, infection with HBV has any impact on disease progression or the development of hepatocellular carcinoma (HCC) in patients with chronic HCV infection. The paper by Marusawa et al (see page 284) has set out to tackle this possibility. They have looked for the presence of anti$\mathrm{HBs}$ and/or anti-HBc in a large cohort of HBsAg negative Japanese patients with HCV infection. The 2014 patients studied were subdivided into three groups-those with chronic hepatitis, those with cirrhosis and those with HCC. In all groups there was a significant increase in the proportion of cases with evidence of previous HBV infection compared with age matched, non-HCV infected controls. Overall, $49.9 \%$ of patients had evidence of previous HBV infection (compared with $12.2 \%$ of controls) with the most striking increase seen in the 611 cases with HCC, of whom over $59 \%$ were anti-HBc positive. Patients with HCC were significantly more likely to have evidence of previous HBV infection than patients with either cirrhosis or chronic hepatitis, and patients with cirrhosis were more likely than those with chronic hepatitis to be anti-HBs and/or anti-HBc positive.

Although the numbers in this study are impressive, the authors have not looked in detail at virological or histological parameters to try and elucidate possible mechanisms for the associations found. It would be interesting to see whether there were any differences in HCV RNA levels or histological activity between patients with and without anti-HBs/anti-HBc, or whether immunostaining or HBV DNA detection from liver biopsy samples revealed evidence of continuing HBV infection. Furthermore, it is not clear how or when the majority of cases studied acquired either infection, so that the role of superinfection (i.e. sequential infection) as opposed to co-infection (simultaneous infection) cannot be answered.

How can a link between previous HBV infection and development of HCC be explained? The association between both chronic $\mathrm{HBV}$ and $\mathrm{HCV}$ infections and development of HCC is well known. Furthermore, there is an increased risk of developing $\mathrm{HCC}$, even in HBsAg negative, anti-HBs positive cases, most probably related to persistence of low levels of hepatic HBV DNA which can also be isolated from tumour tissue. ${ }^{89}$ The HBV encoded $\mathrm{X}$ protein is known to regulate both cell proliferation and apoptosis, and the combination of chronic HCV infection with its attendant increase in hepatocyte turnover together with continuing production of HBV encoded proteins may be synergistic with regard to HCC development.

Is there a link between serological markers of previous HBV infection and disease severity in patients with chronic HCV? The findings reported by Marusawa et al suggest that there is, and this is supported by previous reports. ${ }^{10} \mathrm{~A}$ possible explanation for this is that the onset of $\mathrm{HCV}$ infection occurs after $\mathrm{HBV}$ has been acquired-HBV infection is more prevalent in Japan than in the UK and many of the patients reported on in this series may have acquired $\mathrm{HBV}$ infection vertically and acquired $\mathrm{HCV}$ infection subsequently. HCV superinfection can reduce $\mathrm{HBsAg}$ expression and may promote $\mathrm{HBsAg}$ clearance, ${ }^{4}$ so that anti-HBs/anti-HBc positive patients with $\mathrm{HCV}$ infection may already have had pre-existing chronic liver disease before the onset of their HCV infection.

Do these findings from Japan apply in other countries? The link between previous HBV infection and HCC complicating HCV infection seems clear and has been reported in other populations. ${ }^{9}$ The association between previous $\mathrm{HBV}$ infection and severity of $\mathrm{HCV}$ infection is less clear. In the UK, HBV infection is uncommon and evidence of previous $\mathrm{HBV}$ infection in patients with chronic $\mathrm{HCV}$ is most likely to have resulted from intravenous drug usage, with vertically acquired $\mathrm{HBV}$ being rare. $\mathrm{HBV}$ infection in adults behaves very differently to that in children and further studies are needed to clarify whether apparently resolved HBV infection in adults is truly a factor contributing to the progression of $\mathrm{HCV}$ related liver damage.

M E CRAMP

Institute of Liver Studies,

Kings College Hospital,

London SE5 9RS, UK

1 Niederau C, Lange S, Heintges T, et al. Prognosis of chronic hepatitis C: results of a large prospective cohort study. Hepatology 1998;28:1687-95.

Zarski J-P, Bohn B, Bastie A, et al. Characteristics of patients with dual Zarski J-P, Bohn B, Bastie A, et al. Characteristics of patients
infection by hepatitis B and C viruses. F Hepatol 1998;28:27-33.

3 Sato S, Fujiyama S, Tanaka M, et al. Coinfection of hepatitis C virus in patients with chronic hepatitis B infection. F Hepatol 1994;21:159-66. 
4 Liaw Y-L. Role of Hepatitis C virus in dual and triple hepatitis virus infection. Hepatology 1995;22:1101-8.

5 Rehermann B, Ferrari C, Pasquinelli C, et al. The hepatitis B virus persists for decades after patients' recovery from acute viral hepatitis despite active maintenance of a cytotoxic T-lymphocyte response. Nat Med 1996;2:1104-8.

Hoofnagle J, Seef L, Buskell B, et al. Type B hepatitis after transfuion with blood containing antibody to hepatitis B core antigen. N Engl F Med 1978; 298:1379-83.

7 Chazouilleres O, Mamish D, Kim M, et al. "Occult" hepatitis B virus as source of infection in river transplant recipients. Lancet 1994;343:142-6.
8 Urashima T, Saigo K, Kobayashi S, et al. Identification of hepatitis B virus integration in hepatitis $\mathrm{C}$ virus-infected hepatocellular carcinoma tissues. $\mathcal{F}$ Hepatol 1997;26:771-8.

9 Brechot C, Jaffredo F, Lagorce D, et al. Impact of HBV, HCV and GBV-C/ HGV on hepatocellular carcinomas in Europe: results of a European concerted action. F Hepatol 1998;29:173-83.

10 Villa E, Grottola A, Buttafoc P, et al. Evidence for hepatitis B virus infection in patients with chronic hepatitis $C$ with and without serological markers of hepatitis B. Dig Dis Sci 1995;40:8-13. 